\title{
Engaging community women's groups for improved uptake of antenatal care services in Cross River State, Nigeria: An analysis of post-intervention findings
}

Salisu Mohammed Ishaku

Population Council

Follow this and additional works at: https://knowledgecommons.popcouncil.org/departments_sbsr-rh

Part of the Demography, Population, and Ecology Commons, Family, Life Course, and Society Commons, International Public Health Commons, Maternal and Child Health Commons, and the Women's Health Commons How does access to this work benefit you? Let us know!

\section{Recommended Citation}

Ishaku, Salisu Mohammed. 2018. "Engaging community women's groups for improved uptake of antenatal care services in Cross River State, Nigeria: An analysis of post-intervention findings," Ending Eclampsia Research Brief. Abuja: Population Council. 


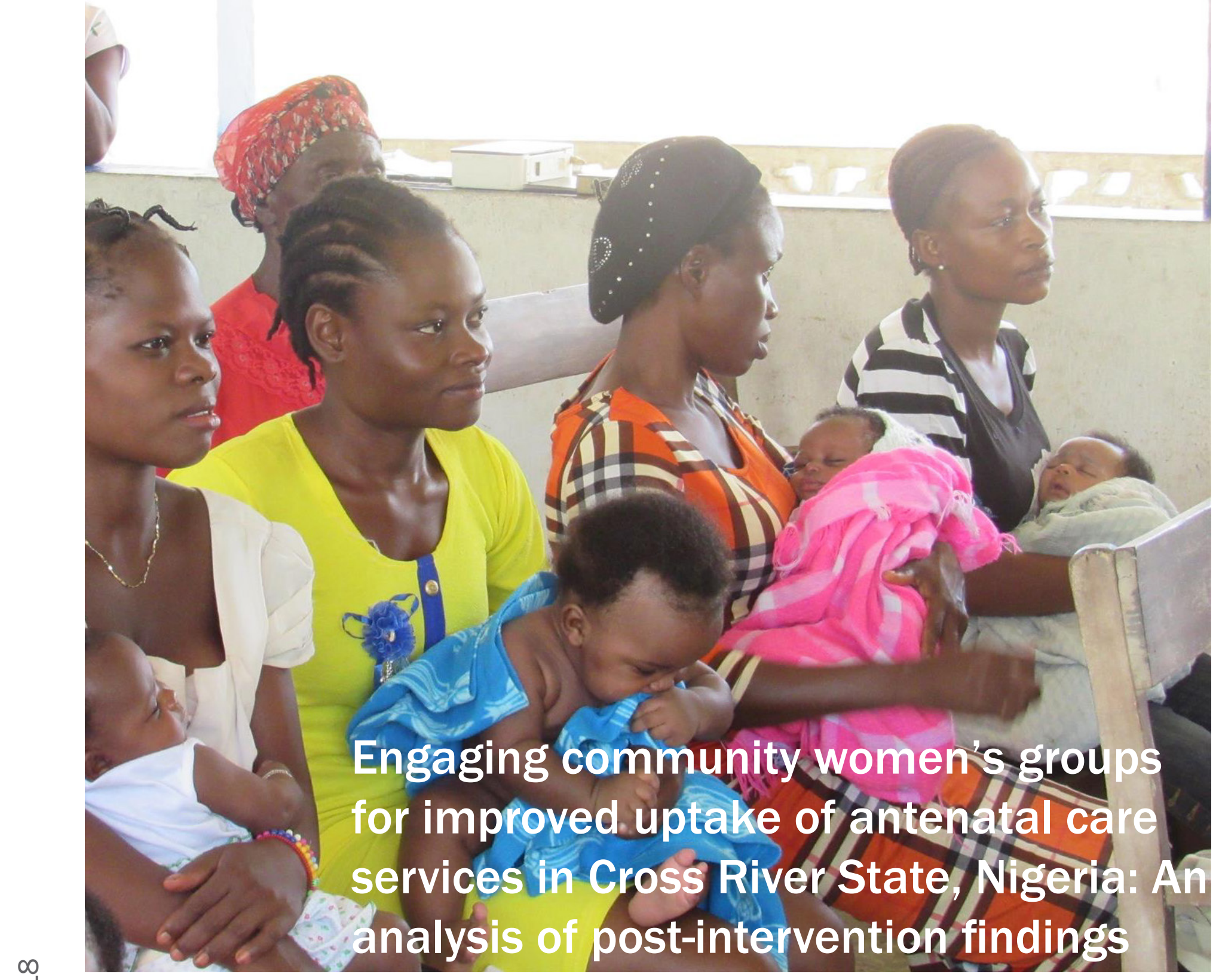




\section{BACKGROUND}

In 2013, pre-eclampsia and eclampsia (PE/E) accounted for one-fifth of all maternal deaths in Nigeria - twice as many deaths as those caused by postpartum hemorrhage. Reports show access to quality maternal health services, including antenatal care (ANC), delivery, and postnatal care (PNC) is low, but there are limited recorded interventions bridging the gap between communities and health providers to improve ANC and reduce preventable deaths from $\mathrm{PE} / \mathrm{E}$.

Some studies report that clients will ask for specific services from their providers - when they know the services exist. Empowering women with the right information about $\mathrm{PE} / \mathrm{E}$ and other hypertensive disorders in pregnancy (HDP) will likely improve access to ANC and improve maternal and newborn health $(\mathrm{MNH})$ outcomes. Preparing women's group leaders to provide important health information to their peers during their community and village group meetings may encourage women to seek services at primary health care $(\mathrm{PHC})$ facilities during pregnancy.

\section{RESEARCH AIM}

The Ending Eclampsia project seeks to understand the potential of underutilized and promising interventions that increase access to services, particularly improving community referral systems. In Nigeria's Cross River State, this study aimed to test the feasibility of women's group leaders delivering health information for PE/E, referral, and other ANC services to their peers, to increasing access to quality MNH services. Post-intervention, researchers conducted in-depth interviews with women's group leaders and women's group members in intervention and comparison communities to determine women's knowledge of PE/E and other HDPs, understand the proportion of women asking for specific services, and assess women's satisfaction with their care. Researchers also conducted focus group discussions (FGDs) with married men and women. Researchers interviewed pregnant women exiting health facilities after ANC visits. Table 1 outlines data collection and activities.

\section{Study Population and Intervention}

Researchers recruited 46 women's group leaders from 24 communities in Cross River and trained them to deliver health education and information to their respective women's groups. Two leaders per group received training on the symptoms and causes of PE/E, hypertension in pregnancy, signs of complications and their implications during pregnancy for women and babies, and the importance of ANC, referral adherence, and follow-up. Leaders received training on services to ask for during ANC/PNC with the intent to empower women so they know what to expect during ANC/PNC consultations. Women's group leaders provided health information during women's meetings, in churches, and through other social gatherings.

\begin{tabular}{|l|c|c|c|c|}
\hline Table 1: Data collection activities \\
\hline & \multicolumn{2}{|c|}{ Intervention } & \multicolumn{2}{c|}{ Comparison } \\
\cline { 2 - 5 } & Baseline & Endline & Baseline & Endline \\
\hline ANC exit interviews & 142 & 53 & 123 & 69 \\
IDIs with women leaders & 16 & 18 & 8 & 10 \\
IDIs with intervention group members & - & 12 & - & - \\
FGDs with community members & - & 2 & - & 2 \\
\hline
\end{tabular}

\section{PE/E IN BRIEF}

- $\quad$ Pre-eclampsia is a condition in pregnant women marked by an increase in blood pressure and protein in urine after 20 weeks gestation.

- Providing high quality antenatal care improves the prevention and early detection of pre-eclampsia and can prevent its progression to eclampsia.

- Eclampsia is a life-threatening condition characterized by convulsions in women with PE.

- Women in developing countries are $\mathbf{3 0 0}$ times more likely to die from eclampsia than women in developed countries.

- $\quad$ Prescribing low-dose aspirin and calcium to at-risk women can prevent pre-eclampsia and eclampsia.

- Pre-eclampsia and eclampsia can be managed by administering anti-hypertensive drugs and magnesium sulphate (MgSO4).

- MgSO4 is the safest and most effective treatment for severe $\mathrm{PE} / \mathrm{E}$, and is one of $13 \mathrm{UN}$ LifeSaving Commodities for Women and Children.

- $\quad \mathrm{PE} / \mathrm{E}$ and other hypertensive disorders in pregnancy increase the risk of pre-term births, which can lead to low birth weight, anemia, and stunting.

- Improved prevention, increased detection, and effective treatment of $\mathrm{PE} / \mathrm{E}$ can prevent unnecessary maternal and newborn deaths.

The Ending Eclampsia project seeks to expand access to proven, underutilized interventions and commodities for the prevention, early detection, and treatment of pre-eclampsia and eclampsia and strengthen global partnerships. 


\section{FINDINGS}

\section{Women's Knowledge and Perceptions of PE/E}

Qualitative data indicate that knowledge of PE/E and its signs and symptoms among women who attended women's groups is mixed. Women's group members know of the possible consequences of high blood pressure (BP) during pregnancy, particularly maternal or fetal death, but misunderstandings of the causes of hypertension and pre-eclampsia exist.

\section{"Pre-eclampsia is caused by lack of adequate diet during pregnancy, lack of money and proper feeding, and because of poor feeding, even drugs cannot work well." \\ "Pre-eclampsia is an illness that causes convulsion and weakens the mother. It could kill the baby, especially when you fall. You could convulse in a way that kills the baby inside you."}

- Women's group members, 2018

While not incorrect, the link between eclamptic convulsions and negative health outcomes without widespread knowledge of the link between hypertension/ pre-eclampsia and eclamptic convulsions suggests a persistent gap in identifying and preventing hypertension and pre-eclampsia early in pregnancy before it progresses to eclampsia and convulsions occur.

Discussions in women's groups covered the importance of ANC registration, how to care for oneself while pregnant, how to prevent infection and malaria, common danger signs during pregnancy, and the importance of delivery in a health facility. In intervention and comparison communities, community members rank malaria as one of the top maternal health challenges, while hypertension

Figure 1: Women's knowledge of PE/E (\%)

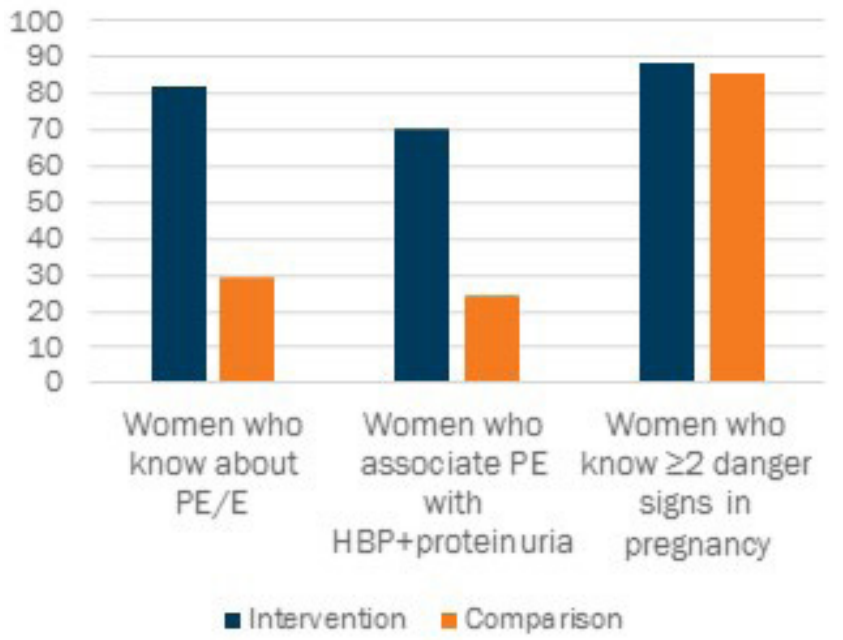

and PE/E are infrequently mentioned. Evidence from exit interviews with women who attended ANC in both intervention and comparison communities shows the efficacy of incorporating health education about PE/E into women's group activities. Figure 1 demonstrates that, while women across both groups know of several danger signs in pregnancy, more women in the intervention group associate high BP and proteinuria with PE/E. Women also associate swelling and headache with high BP, and participants readily seek care when symptoms occur.

Community engagement is high in Cross River State. Nearly all women from intervention and comparison communities suggest working with town criers, churches, and women's and men's groups for general and pregnancy-specific health education.

\section{Patient-Provider Interactions and Women's ANC Experiences}

Women's group members seek health services at hospitals and PHCs. Many women know PHCs may refer them to secondary facilities, which prompts them to bypass PHCs and first visit a hospital for care. Most women expect to receive $\mathrm{BP}$ checks and abdominal palpations during ANC and few women are willing and able to ask providers about specific ANC services if they don't receive them. Most women had their BP and urine checked, but more women in the intervention group had their results explained. Women consistently report being treated well at PHCs and hospitals, though they seek care from hospitals rather than from PHCs due to perceived insufficient number of providers and lack of worker motivation at PHCs. Figure 2 on the following page details women's reported experiences during ANC.

\section{Member Experiences with Women's Groups}

Women enjoy attending women's groups and find the provided information useful. Women leaders are trustworthy figures, often relied on for support regarding pregnancy-related complications. Women's groups in intervention and comparison communities play an important role in encouraging women to seek health and ANC services.

Though participants' recognition of PE/E terminology remains thin, there is increasing awareness of the importance of ANC registration and seeking facility-based care for pregnancy-related complications, including PE/E symptoms. Women's group leaders teach members that swollen legs and convulsions are signs of high BP and they should seek treatment at health facilities for healthy birth outcomes. 
Figure 2: Women's experiences during ANC (\%)

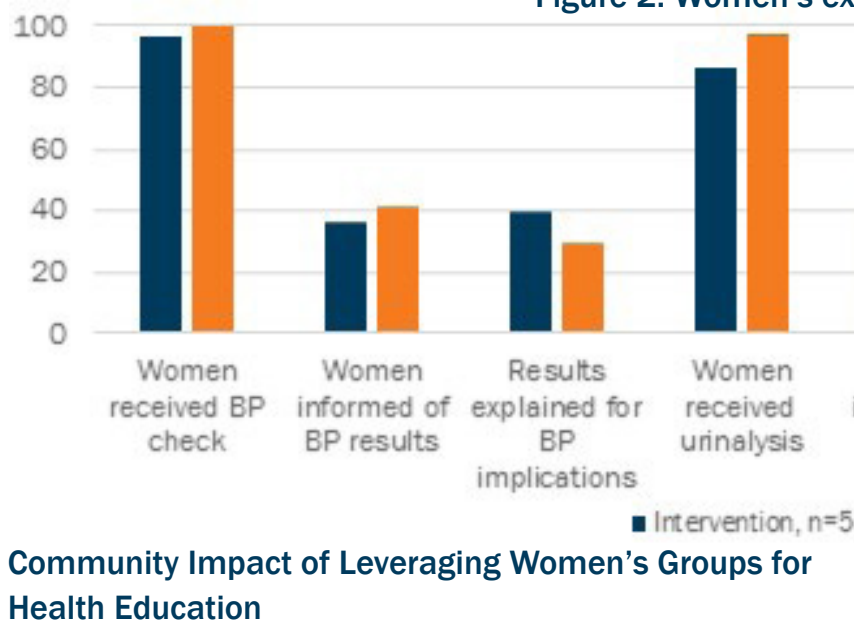

In communities where women's group leaders provide health education, entire communities-men and womenare mobilized to encourage pregnant women's facilitybased health-seeking behaviors. At baseline, religious leaders were said to primarily pray for a woman and bring her into the church to deliver, but FGD participants now report that religious leaders encourage women to seek medical care when complications occur. It is clear from FGDs that prayer and church community continue to be powerful resources for women during pregnancy, but now co-exist with a woman's facility-based care.

\section{"Communities see such a thing (maternal death) and they are not happy... That's why they push women, they support women and help them with maternity care, they join together." \\ - Woman leader, Intervention community}

Women's group leaders from comparison communities individually encourage women to register for ANC services and seek health services from PHCs or hospitals, but state that religious leaders and other community members are more likely to promote traditional health practices such as rubbing kernel oil on the body. Traditional beliefs of the causes of high BP persist among women throughout Cross River State and include over-thinking and headache, though attributions of witchcraft were more likely to be seen in non-intervention communities.

Additionally, in intervention communities, PHCs are more likely to be the first place a woman seeks care, whereas in non-intervention communities, women's group leaders encourage women to seek care directly from the hospital.

\section{DISCUSSION}

Women's groups' findings in Cross River suggest that community engagement is a critical model for

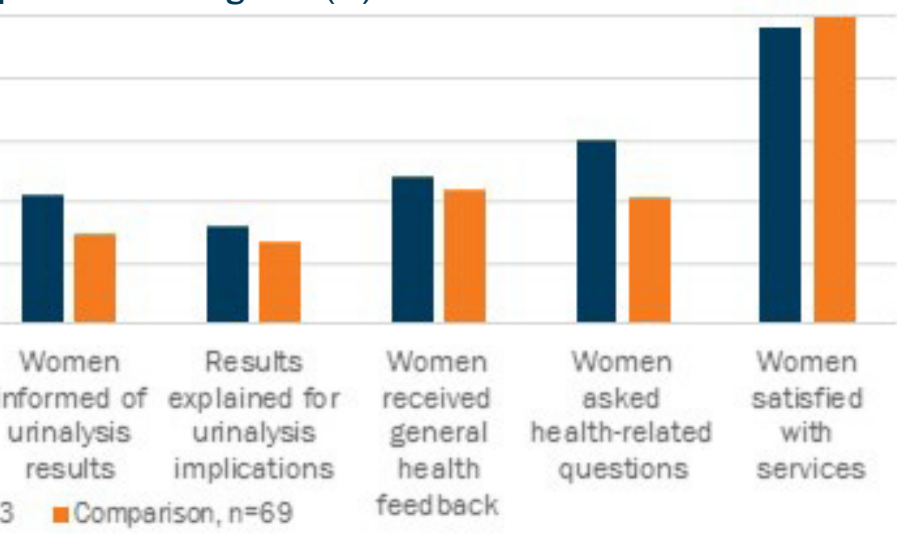

information sharing and is an adaptable, acceptable model for increasing PE/E prevention and danger sign recognition across Nigeria. Qualitative findings reveal that participants and leaders across intervention and comparison communities emphasize incorporating PE/ E-related health education into socioculturally relevant community groups. Other context-based solutions emerged: Involving the town crier as a facilitator to encourage women to register for ANC and to visit PHCs for pregnancy-related complications.

Following the implementation of women's groups activities, knowledge of hypertension and pregnancy complications increases alongside awareness of the importance of ANC attendance at health facilities. While symptoms of eclampsia (convulsions) are understood, gaps persist in the recognition of signs of hypertension/ $\mathrm{PE} / \mathrm{E}$, as well as in prevention strategies.

\section{RECOMMENDATIONS}

- Sustain current levels of training and information support to community women's groups to provide community health education;

- Improve training for women's group leaders to better disseminate information on the warning signs and symptoms of hypertension and pre-eclampsia, as well as prevention strategies;

- Empower women to continue their health seeking behaviors through community education on hypertension and $\mathrm{PE} / \mathrm{E}$ to better recognize signs and symptoms of each; and

- Develop institutional guidance at national level for facilitating community-facility linkages with key stakeholders as mobilizers of effective care-seeking for hypertension-related complications during pregnancy.

\section{Contact}

Salisu Mohammed Ishaku

Reproductive Health Program Director, Population Council, Nigeria

Suggested Citation

Salisu Mohammed Ishaku. 2018. “Engaging community women's groups for improved uptake of antenatal care services in Cross River State, Nigeria: An analysis of post-intervention findings," Ending Eclampsia Research Brief. Abuja: Population Council. 